\title{
Problemática en la determinación de módulo elástico y dureza de materiales cerámicos de alta rigidez mediante indentación
}

\author{
A. RICO, M. A. GARRIDO, J. RODRÍGUEZ
}

Departamento de Ciencia e Ingeniería de Materiales. Universidad Rey Juan Carlos, 28933 Móstoles (Madrid).

\begin{abstract}
En este trabajo se han realizado ensayos de indentación instrumentada sobre dos materiales cerámicos empleando indentadores Berkovich y esférico a diferentes cargas máximas. Se ha determinado su dureza y el módulo de elasticidad a partir del análisis de las curvas carga - desplazamiento con el método de Oliver y Pharr. Se ha observado que los valores de ambas propiedades cambian con el tamaño de la indentación. Sin embargo, estas variaciones dependen de diversos factores como el efecto del tamaño de la indentación, la rigidez del material indentado y la geometría del indentador. Con el fin de acotar el efecto que la deformación del indentador tiene sobre las tendencias observadas, se han realizado simulaciones por elementos finitos del ensayo de indentación sobre un material elástico lineal con las propiedades del material cerámico más rígido estudiado (carburo de Boro) y analizando los casos de indentador rígido y elástico.
\end{abstract}

Palabras clave: Nanoindentación, propiedades mecánicas, materiales cerámicos

The problem of determining Young's modulus and hardness of high stiff ceramics by nanoindentation

Sensing indentation tests at different maximum loads were carried out on two crystalline ceramic materials using Berkovich and spherical indenters. Hardness and Young's modulus were estimated from load - displacement curves through Oliver and Pharr method. Values from mechanical properties change with the penetration depth depending on indentation size, material stiffness and geometry of the indenter. To estimate the indenter deformation effect, finite elements simulations were developed to simulate the indentation process on an elastic material with the properties of the stiffer material (Boron carbide).

Keywords: Nanoindentation, mechanical properties, ceramic materials.

\section{INTRODUCCIÓN}

Las técnicas de indentación instrumentada son adecuadas para estimar las propiedades mecánicas de pequeños volúmenes de material. Por ello, se han utilizado ampliamente en la estimación de propiedades tales como módulo elástico y dureza en recubrimientos de todo tipo, desde metálicos hasta cerámicos. Sin embargo, este tipo de técnicas presentan una serie de particularidades que exigen prestar una cuidada atención a los datos numéricos que se extraen de los ensayos.

La técnica consiste en registrar simultáneamente la profundidad y la fuerza que experimenta un indentador de geometría y propiedades conocidas cuando penetra en un material cuyas propiedades se desean obtener.

La figura 1 recoge una curva fuerza - desplazamiento experimental obtenida a partir de una indentación sobre nitruro de aluminio utilizando un indentador Berkovich. Del análisis de este tipo de curvas, es posible obtener el módulo elástico y la dureza del material desconocido sin tener que medir la huella residual, al contrario que en los métodos de indentación tradicionales. El método de cálculo que se emplea tradicionalmente para determinar estas propiedades se basa en los estudios realizados por Oliver y Pharr (1), que a su vez se basaron en los trabajos desarrollados por Hertz, Sneddon, Doerner y Nix $(2-4)$ acerca del contacto elástico entre un indentador de geometría conocida y un plano elástico.

Si un indentador esférico penetra en un material elástico,



Fig. 1- Curva experimental fuerza - desplazamiento obtenida a partir de una indentación sobre nitruro de aluminio con un indentador Berkovich. En la figura se representa: carga máxima de indentación $\left(\mathrm{P}_{\max }\right)$, penetración máxima de indentación $\left(\mathrm{h}_{\mathrm{t}}\right)$, penetración de contacto $\left(h_{\mathrm{p}}\right)$, penetración residual $\left(\mathrm{h}_{\mathrm{r}}\right)$ y pendiente de la rama de descarga evaluada a penetración máxima (S). 
la relación entre la carga, $\mathrm{P}$, aplicada y el desplazamiento elástico, $\mathrm{h}_{\mathrm{e}^{\prime}}$ producido puede expresarse como (2):

$$
P=C \cdot h_{e}^{3 / 2}
$$

Sin embargo, si se emplea un indentador cónico, esta relación cambia a (3):

$$
P=C \cdot h_{e}^{2}
$$

En ambos casos, la constante $\mathrm{C}$ depende de la geometría del indentador y de sus propiedades mecánicas, así como de las propiedades mecánicas de la muestra.

Según el método de Oliver y Phar (1), la pendiente de la rama de descarga, $\mathrm{S}$, de la curva $\mathrm{P}-\mathrm{h}$, se mide a carga máxima y permite obtener un módulo reducido, $\mathrm{E}^{*}$, utilizando el área de contacto, A:

$$
S=\frac{2}{\sqrt{\pi}} \cdot \frac{1}{\beta} \cdot E^{*} \cdot \sqrt{A}
$$

Donde $\beta$ es un factor corrector estimado mediante elementos finitos, y que vale 1,034 en el caso de que se utilice un indentador de geometría Berkovich, y 1 en el caso de que se emplee un indentador esférico.

El área de contacto entre el indentador Berkovich y la muestra en la situación de máxima carga, puede calcularse a partir de la expresión:

$$
A=24.5 \cdot h_{p}{ }^{2}=24.5 \cdot\left[h_{t}-\frac{2 \cdot(\pi-2)}{\pi} \frac{P_{\max }}{S}\right]^{2}
$$

En el caso de un indentador esférico, el área de contacto se puede estimar con:

$$
A=\pi \cdot\left(2 \cdot R_{i} \cdot h_{p}\right)=\pi \cdot 2 \cdot R_{i} \cdot\left(h_{t}-\frac{3}{4} \frac{P_{\max }}{S}\right)
$$

Donde $h_{p}$ y $h_{t}$ son la profundidad de contacto y la profundidad total respectivamente. $\mathrm{R}_{\mathrm{i}}$ es el radio del indentador esférico. Una vez que se estima el módulo compuesto, es posible relacionarlo con el módulo elástico a través de la expresión:

$$
\frac{1}{E^{*}}=\frac{1-v^{2}}{E}+\frac{1-v_{i}^{2}}{E_{i}}
$$

Donde $\mathrm{E}, \mathrm{E}_{\mathrm{i}}, v$ y $v_{\mathrm{i}}$ son el módulo elástico y el coeficiente de Poisson de la muestra y del indentador, respectivamente.

Por último, la dureza del material puede calcularse como:

$$
H=\frac{P_{\text {max }}}{A}
$$

La precisión del método depende de la capacidad para determinar adecuadamente el área de contacto entre el indentador y la muestra. Por ello, si existen desviaciones en la geometría real del indentador con respecto a la ideal considerada en el método de Oliver y Pharr se cometerán errores en el cálculo de las propiedades mecánicas de la muestra. Por otro lado, existen diversos efectos asociados al tamaño de la indentación que se manifiestan, en gran medida, en la escala nanométrica. Algunos de ellos, están relacionados con la respuesta del propio material, sin embargo, hay otros que pueden deberse a artefactos introducidos por el propio método de análisis de las curvas fuerza - desplazamiento. A continuación se revisan los más importantes:

- Efecto del tamaño de la indentación: La dureza medida mediante técnicas de indentación instrumentada con indentadores agudos (como Berkovich o Vickers) presenta, en algunos materiales cristalinos, una fuerte dependencia con la profundidad de penetración, es decir, con el tamaño de la indentación. Normalmente, la dureza aumenta a medida que disminuye la profundidad de penetración. Esta dependencia se ha intentado explicar desde diferentes puntos de vista, siendo uno de los más aceptados el propuesto por Nix y Gao $(5-7)$, que basa la explicación para esta variación de la dureza en la formación de dislocaciones geométricamente necesarias en el entorno de la indentación, debido al elevado gradiente de deformaciones que provoca un indentador de este tipo cuando penetra en el material. Estas dislocaciones se añaden a las ya existentes generadas por el propio proceso de deformación, denominadas dislocaciones estadísticamente almacenadas, provocando un efecto de endurecimiento adicional que es introducido por el propio método de medida. Según el modelo propuesto, la dureza varía con la profundidad de penetración según la siguiente expresión:

$$
\frac{\mathrm{H}}{\mathrm{H}_{0}}=\sqrt{1+\frac{\mathrm{h}^{*}}{\mathrm{~h}_{\mathrm{t}}}}
$$

Donde $\mathrm{H}_{0}$ es la dureza del material que se obtendría en

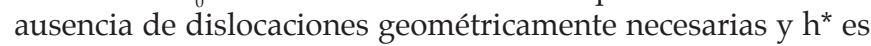
un factor de escala.

La dureza medida utilizando indentadores esféricos también puede presentar un efecto del tamaño de la indentación, asociado a la transición entre el régimen elástico y el elasto - plástico que se produce en el material a medida que la carga de indentación aumenta. Esta transición es debida a que esta geometría no presenta similitud geométrica (8).

- Desviaciones en la geometría de la punta:

Las desviaciones que presenta el indentador en su geometría con respecto a la geometría ideal que se asume en el modelo de Oliver y Pharr, pueden producir inexactitudes en la estimación de las propiedades mecánicas a través de un ensayo de indentación instrumentada. Por ejemplo, en el caso de indentadores como Berkovich o Vickers, y debido a la gran dificultad que implica la fabricación de este tipo de dispositivos, existe un redondeo en su punta, que no se considera en el modelo de Oliver y Pharr. Las simulaciones mediante elementos finitos que llevaron a cabo $\mathrm{Yu}$ et al (9), demuestran que el efecto que tiene este redondeo sobre la dureza es similar al que se produce como consecuencia del tamaño de la indentación descrito anteriormente. Es decir, a medida que disminuye el tamaño de la indentación la dureza aumenta. Por tanto, ambos efectos pueden llegar a confundirse. Alkorta et al. han incluido recientemente este fenómeno en un modelo basado en el propuesto por Nix y Gao (10).

Deformación del indentador:

Cuando el material de prueba es muy rígido, como es el caso de los materiales cerámicos, el indentador puede llegar a deformarse provocando una variación en la geometría del mismo con respecto a la geometría ideal que se utiliza en el modelo de Oliver y Pharr. Knapp et al. y Faulkner et al. (11 - 13) determinaron, utilizando simulaciones de elementos finitos, que la deformación del indentador puede producir 
grandes errores en la determinación de las propiedades mecánicas cuando no se tiene en cuenta.

Rugosidad de la superficie:

Las ecuaciones desarrolladas en el modelo de Oliver y Pharr, se basan en las ecuaciones del contacto elástico entre un indentador de geometría conocida, y un plano elástico. Por tanto, si la rugosidad de la superficie del material que se pretende ensayar es elevada, esta suposición del modelo no se verifica. Rico et al. (14) concluyeron que la dureza y módulo elástico determinados mediante nanoindentación disminuyen a medida que aumenta la rugosidad de la superficie, de modo que sólo es posible obtener valores adecuados de esta propiedades mecánicas cuando el cociente entre la rugosidad y la profundidad de penetración se encuentra por debajo de un valor crítico que depende de cada material.

Es necesario indicar que todos estos efectos que distorsionan el valor de las propiedades mecánicas que se pretenden conocer mediante el ensayo de indentación instrumentada son más significativos en la escala nanométrica, de modo que afectan más a aquellos materiales que presentan una rigidez elevada, pues es difícil alcanzar grandes penetraciones durante el ensayo.

Todos los fenómenos descritos anteriormente conducen a una estimación errónea del área de contacto entre el indentador y la muestra. Puesto que la dureza y el módulo elástico dependen de esta variable según las ecuaciones [3] y [7], también se comete error en la estimación de estas propiedades.

Como demostraron Guicciardi et al. y Gong et al. (15 - 18) las curvas $\mathrm{P}-\mathrm{h}$ obtenidas del ensayo de nanoindentación en materiales cerámicos puede verse afectada por la microestructura del material.

Este trabajo trata de poner de relieve la problemática existente cuando se indentan materiales cerámicos en la escala nanométrica. Para ello, se han ensayado diferentes materiales cerámicos policristalinos con indentadores de geometría Berkovich y esférica. Con el fin de minimizar los efectos geométricos, se propone una metodología basada en el empleo de ensayos a diferentes cargas máximas de indentación. Para apoyar algunas de las conclusiones alcanzadas a partir del estudio experimental, se han realizado simulaciones de elementos finitos del proceso de indentación en el material más rígido (Carburo de Boro).

\section{MATERIALES}

Se han ensayado dos materiales cerámicos policristalinos: Nitruro de Aluminio y Carburo de Boro, que fueron suministrados por Morgan Matroc $^{\mathrm{TR}}$. Las principales propiedades mecánicas para cada uno de estos materiales se muestran en la tabla 1. En el caso del diamante, se indican como propiedades mecánicas las que ha proporcionado el

TABLA I. PROPIEDADES MECÁNICAS DE LOS MATERIALES INVOLUCRADOS EN EL ESTUDIO. SE HA INDICADO ENTRE PARÉNTESIS LA DESVIACIÓN ESTÁNDAR DE LOS VALORES OBTENIDOS EXPERIMENTALMENTE.

\begin{tabular}{|l|c|c|c|}
\hline Material & $\begin{array}{c}\text { Nitruro de } \\
\text { Aluminio }\end{array}$ & $\begin{array}{c}\text { Carburo de } \\
\text { Boro }\end{array}$ & Diamante \\
\hline $\begin{array}{l}\text { Módulo de Young } \\
(\mathrm{GPa})\end{array}$ & $340(16)$ & $460(1,2)$ & 1140 \\
\hline Dureza Vickers (GPa) & $12(0,8)$ & $34(1,6)$ & 85 \\
\hline Coeficiente de Poisson & 0,25 & 0,20 & 0,07 \\
\hline Densidad relativa (\%) & 98,0 & 99,7 & - \\
\hline
\end{tabular}

fabricante de los indentadores empleados (MTS $\left.{ }^{\mathrm{TR}}\right)$. Se han realizado medidas experimentales de dureza Vickers con carga de $3 \mathrm{~N}$ sobre ambos materiales y se ha medido la densidad relativa de los materiales involucrados. Los valores de módulo elástico se han obtenido mediante ensayos de propagación de ondas elásticas sobre ambos materiales (19). Cuando se dispone de ello, se incluye una estimación de la dispersión de los resultados.

\section{METODOLOGÍA EXPERIMENTAL}

Las muestras se pulieron con el fin de eliminar la posible rugosidad superficial de las mismas. En la última etapa de pulido se utilizó pasta de diamante con un tamaño de abrasivo de $0,25 \mu \mathrm{m}$.

Se emplearon dos geometrías para el indentador: Una punta Berkovich con un radio nominal en el vértice de 100 $\mathrm{nm}$, y un indentador esférico de $4,9 \mu \mathrm{m}$ de radio. El equipo experimental utilizado es un nanoindentador XP de MTS. La carga se aplica mediante una bobina electromagnética calibrada con una resolución de $50 \mathrm{nN}$. El desplazamiento que experimenta el indentador se mide mediante un transductor capacitivo con una resolución de $0,01 \mathrm{~nm}$. Se realizaron 20 series de indentación con 10 indentaciones en cada una de ellas, variando la carga máxima de indentación entre 1 y 500 $\mathrm{mN}$ para los materiales involucrados en el estudio. Antes de realizar los ensayos, se realizó una calibración del indentador utilizando sílice como patrón.

\section{SIMULACIÓN NUMÉRICA}

Con el fin de determinar los posibles efectos de la deformación del indentador durante un ensayo de indentación, se ha simulado una indentación elástica sobre un material elástico cuyas constantes elásticas coinciden con las del carburo de boro, empleando el código de elementos finitos ANSYS.

El indentador se ha modelizado mediante un cono con un área equivalente a la del indentador Berkovich, con un radio final de acuerdo de $100 \mathrm{~nm}$, según las dimensiones nominales proporcionadas por el fabricante.

Empleando las capacidades de axisimetría del problema, tanto el indentador como la muestra se han simulado mediante superficies de revolución bidimensionales.

Se ha escogido un elemento de ocho nodos bidimensional de alto orden con dos grados de libertad en cada nodo (traslaciones en las direcciones $\mathrm{x}$ e $\mathrm{y}$ ) para modelizar tanto al indentador como al material indentado, con elementos de contacto compatibles entre ambas superficies.

En la figura 2 se muestra el modelo y mallado utilizado. Los nodos pertenecientes al eje de simetría sólo pueden desplazarse a lo largo del mismo. Con el fin de reproducir un ensayo de indentación, los nodos situados en la parte inferior, y que limitan el material a indentar, tienen impedido los desplazamientos verticales.

Una vez definido el modelo y las condiciones de contorno, el código de elementos finitos debe alimentarse con las propiedades de los materiales. El indentador es elástico con los valores de las propiedades del diamante: módulo elástico de 1140 GPa y coeficiente de Poisson de 0,07. El material indentado también es elástico con las propiedades nominales 

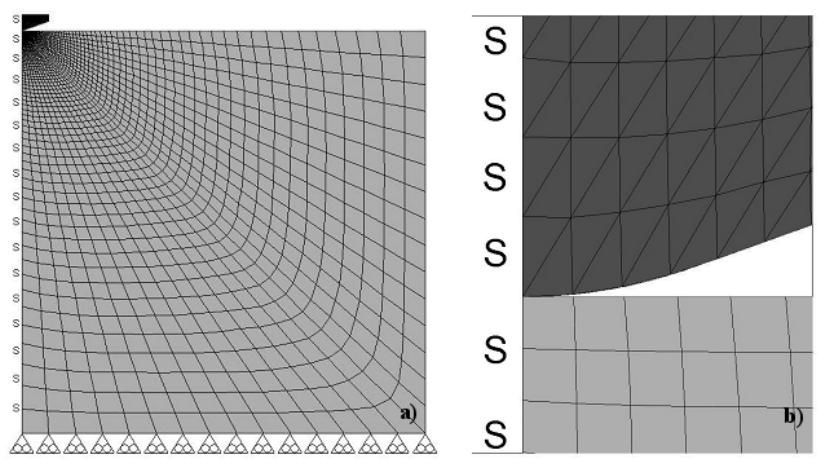

Fig. 2- Esquema del modelo de elementos finitos empleado. a) Esquema general de la geometría y mallado impuesto. b) Detalle del contacto entre indentador y material indentado.

del carburo de boro: módulo elástico de 460 GPa y coeficiente de Poisson de 0,20 .

Para simular un proceso de indentación, se impone un desplazamiento de $1000 \mathrm{~nm}$ sobre el indentador, registrando durante todo el proceso tanto el desplazamiento vertical que experimentan los nodos del indentador en contacto con la superficie indentada, como la correspondiente fuerza, que se obtiene sumando las reacciones sobre los nodos inferiores.

\section{RESULTADOS Y DISCUSIÓN}

La figura 3 muestra el valor de las propiedades mecánicas obtenidas para cada uno de los materiales estudiados, representados frente a la profundidad de penetración total, para los dos indentadores empleados. Tanto el módulo elástico como la dureza presentan una fuerte dependencia con el tamaño de la indentación, si bien las tendencias que se observan varían en función del indentador que se utiliza. El módulo elástico aumenta a medida que disminuye el tamaño de la indentación. Esta tendencia se verifica tanto para la punta Berkovich como para el indentador esférico. Sin embargo, en el caso de la dureza se observa que cuando se utiliza el indentador Berkovich sigue la misma tendencia que la descrita para el caso del módulo, pero se invierte
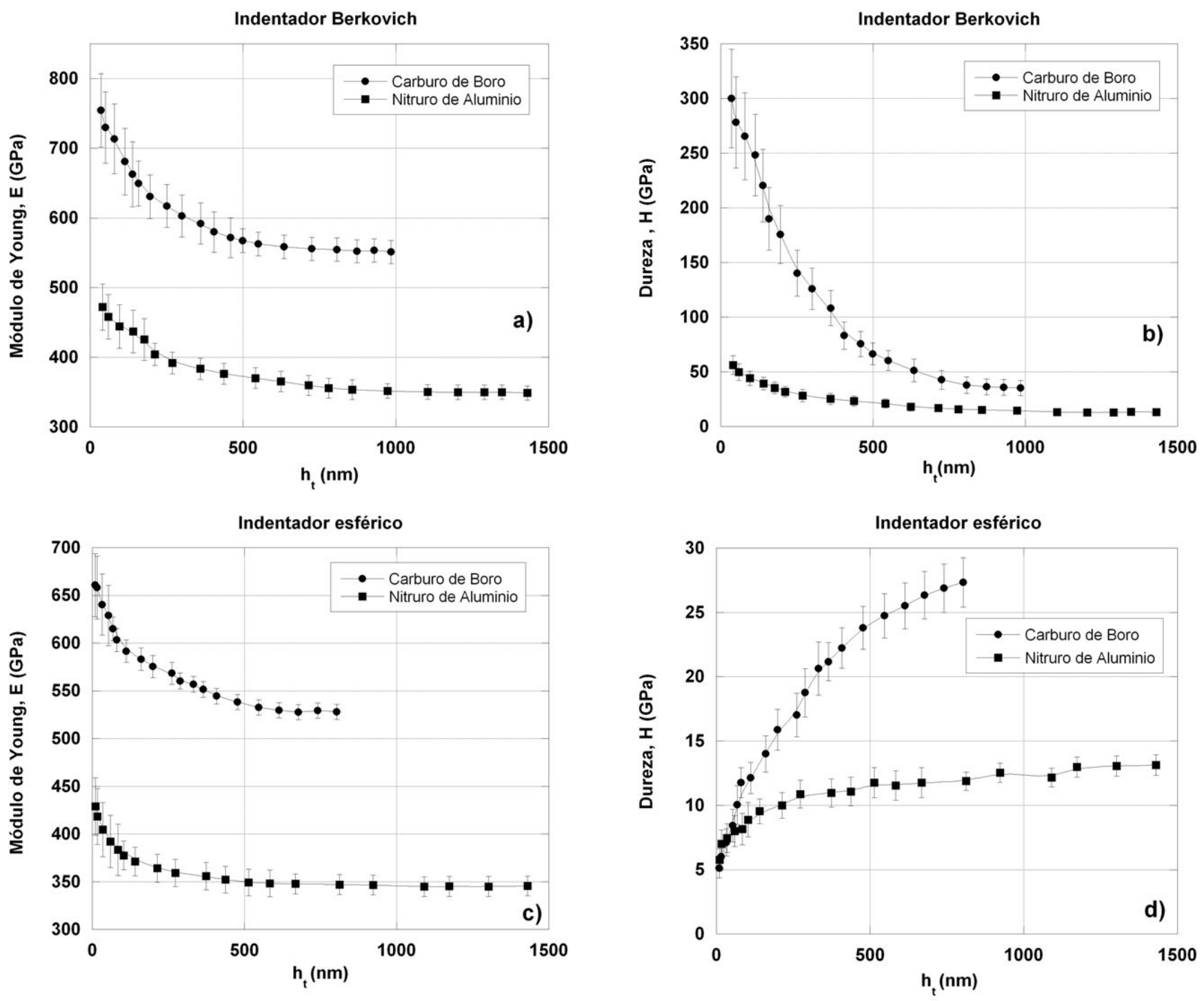

Fig. 3- Valores de las propiedades mecánicas de los materiales estudiados obtenidos experimentalmente frente a la profundidad de penetración. a) Módulo elástico obtenido de ensayos realizados con indentador Berkovich. b) Dureza obtenida de ensayos realizados con indentador Berkovich. c) Módulo elástico obtenido de ensayos realizados con indentador esférico. d) Dureza obtenida de ensayos realizados con indentador esférico. 
cuando se realiza el ensayo con una punta esférica. Es decir, la dureza aumenta a medida que aumenta la profundidad de penetración. De igual modo, se puede comprobar que tanto la dureza como el módulo elástico tienden hacia un valor asintótico cuando la profundidad de penetración es elevada. En el nitruro de Aluminio esta asíntota coincide con el valor nominal, mientras que en el carburo de Boro, este valor es superior al nominal.

En una primera aproximación, la variación de la dureza puede explicarse atendiendo al modelo de Nix y Gao (5) cuando se utiliza un indentador Berkovich, y a una transición entre el régimen elástico y el elasto - plástico en el caso de emplear un indentador esférico (8). Además, Swadener et al. (20) demostraron que la dureza medida con indentadores esféricos puede depender del radio del indentador. Sin embargo, el módulo de Young no depende de fenómenos asociados a la generación de dislocaciones, o de transiciones entre regímenes, ya que esta variable se encuentra asociada a propiedades mucho más básicas.

La dureza y el módulo de Young obtenidos mediante indentación están relacionados con el área de contacto entre el indentador y la muestra, según el método de Oliver y Pharr, a través de las ecuaciones [3] y [7], por tanto, aquellos factores que afecten a la estimación de esta área de contacto afectarán también al módulo elástico y a la dureza.

Tradicionalmente, para evitar el error en la estimación del área de contacto se suele hacer una calibración con un material patrón. Esta calibración permite obtener una relación matemática entre el área de contacto y la penetración de contacto que tienen en cuenta que la geometría del indentador no es ideal. Sin embargo, cuando la rigidez del patrón es muy diferente de la rigidez de las muestras que se van a ensayar, la función obtenida no se puede aplicar.

En la tabla 1 se aprecia como el módulo elástico de los materiales involucrados no es despreciable con respecto al del diamante. Por tanto durante el proceso de indentación el indentador puede llegar a deformarse. Puesto que el área de contacto cuando el indentador se encuentra deformado es mayor que el área que se estima con el método de Oliver y Pharr, las propiedades mecánicas se sobrevaloran.

Con el fin de apoyar esta hipótesis se ha realizado una simulación mediante elementos finitos del proceso de indentación sobre un material elástico con las constantes elásticas del Carburo de Boro, el cerámico más rígido estudiado. Se ha comparado el caso en el que el indentador es rígido, con el caso en el que el indentador es elástico con las propiedades del diamante. En la figura 4, se compara el perfil de la geometría del indentador en la situación de máxima carga para el indentador rígido y elástico. Como se puede comprobar, en el caso elástico, el indentador sufre una deformación considerable, que apoya la hipótesis expresada anteriormente. Para reforzar aún más este concepto, en la figura 5 se presenta una comparación entre la curva $\mathrm{P}-\mathrm{h}$ obtenida a partir de un nodo que se encuentra en la superficie superior del indentador, y la curva $\mathrm{P}$ - h estimada a partir de los desplazamientos sufridos por un nodo que se encuentra en la punta del indentador. Como se puede comprobar, aunque la fuerza que experimentan los dos nodos es la misma, mientras que el nodo superior baja los $1000 \mathrm{~nm}$ impuestos en la simulación, el nodo inferior sólo alcanza los $760 \mathrm{~nm}$, lo cual indica que el indentador se está deformando. Si bien este resultado reafirma la hipótesis emitida acerca del error cometido en la estimación del área de contacto debido a la

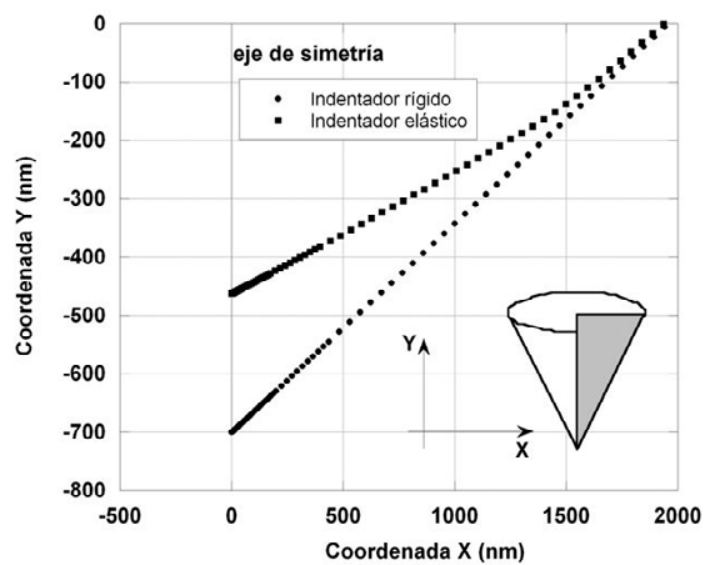

Fig. 4- Comparación entre los perfiles de la geometría del indentador elástico y rígido, obtenidos mediante simulación por elementos finitos. Se presenta un esquema de la sección del indentador representado en la gráfica.

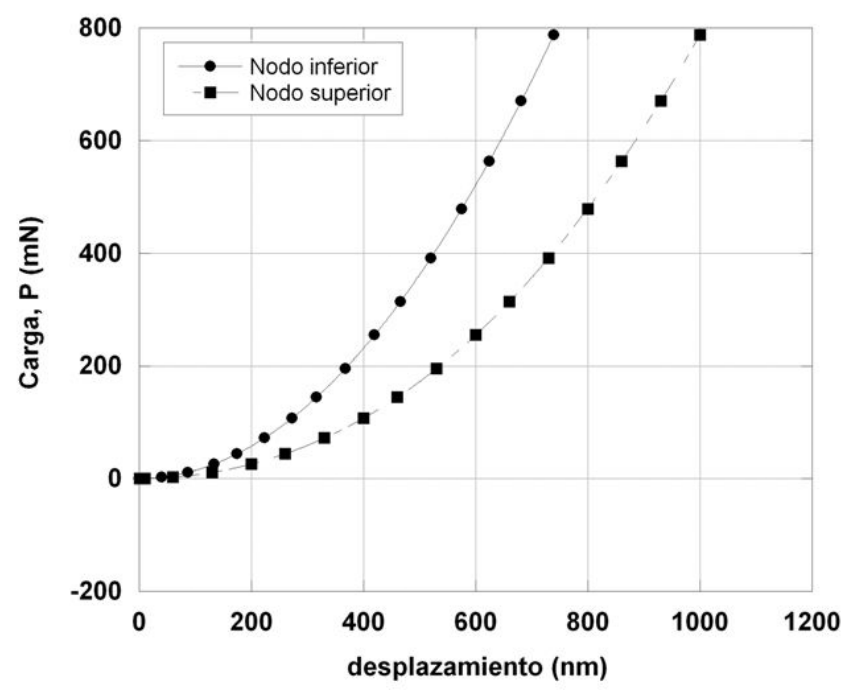

Fig. 5- Comparación entre las curvas $\mathrm{P}$ - h de un nodo que se encuentra en la superficie superior del indentador y un nodo que se encuentra en la punta del mismo, obtenidas a partir de simulaciones por elementos finitos.

deformación del indentador, en la situación real ha de tenerse en cuenta que se pueden producir fenómenos de plastificación no contemplados en esta simulación.

Los fenómenos que se han descrito anteriormente provocan una estimación errónea del área de contacto cuando se emplea el método de Oliver y Pharr para analizar las curvas $\mathrm{P}-\mathrm{h}$. Con el fin de minimizar estos efectos geométricos, se propone una metodología basada en la utilización de múltiples ensayos para determinar las propiedades mecánicas del material cerámico que se indenta. En la figura 6 a se representa la pendiente de la rama de descarga evaluada a carga máxima, S, frente a la penetración de contacto, $h_{\mathrm{p}^{\prime}}$ medidas de los ensayos realizados con la punta Berkovich. De acuerdo con las ecuaciones [3] y [4], la pendiente de esta representación está relacionada con el módulo compuesto $E^{*}$. Como se muestra en la figura, el coeficiente de correlación que se obtiene tras realizar un ajuste lineal sobre los datos es próximo a 1 . Una vez determinado el módulo compuesto a partir de la pendiente, es posible obtener el módulo de Young, a través de la ecuación [6].

Se puede aplicar el mismo tipo de análisis a los ensayos realizados con punta esférica sin más que representar $S$ vs. $\mathrm{h}^{1 / 2}$ (figura $6 \mathrm{~b}$ ). A partir de la pendiente de este gráfico 
es posible obtener, de nuevo, el módulo combinado $\mathrm{E}^{*}$, tal y como indican las ecuaciones [3] y [5]. El coeficiente de correlación lineal obtenido del ajuste presenta un buen valor. Posteriormente es posible determinar el módulo de Young del material utilizando la ecuación [6]. En la tabla 2 se indican los resultados obtenidos para cada material y para los dos indentadores utilizados.
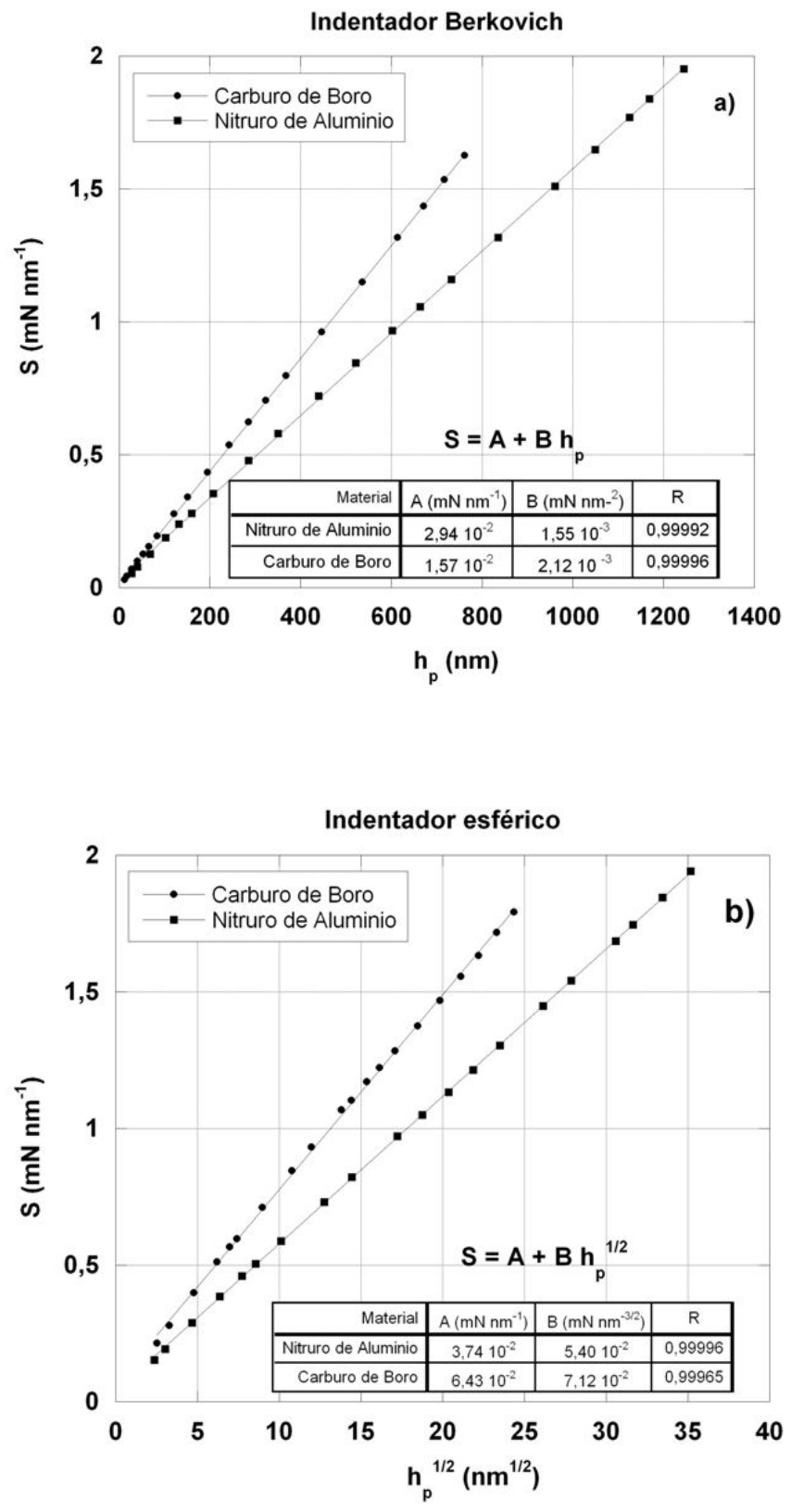

Fig. 6- a) Pendiente de la rama de descarga, S, frente a la profundidad de contacto, $h_{p^{\prime}}$ para los dos materiales estudiados ensayados con indentador Berkovich. b) Pendiente de la rama de descarga, S, frente a la raíz cuadrada de la profundidad de contacto, $h_{p^{\prime}}$ para los dos materiales estudiados ensayados con indentador esférico.

Los valores de módulo elástico obtenidos del ajuste son muy similares a la asíntota a la que tienden los valores experimentales recogidos en las figura $3 a$ y $3 b$. La ventaja de este método se encuentra en que permite obtener este valor sin necesidad de alcanzar la asíntota experimentalmente, ya que el ajuste lineal puede realizarse sobre un número inferior de datos experimentales.

De la tabla 2, se puede concluir que el material más rígido (carburo de Boro) presenta un módulo de Young superior al nominal expresado en la tabla 1. Por otro lado, el nitruro de Aluminio presenta un valor del módulo de Young calculado a partir del ajuste lineal similar al nominal.

TABLA II. MÓDULO ELÁSTICO Y DUREZA DE LOS MATERIALES OBTENIDOS A PARTIR DE LOS AJUSTES DE LAS FIGURAS 4 Y 5.

\begin{tabular}{|c|c|c|c|c|}
\hline & \multicolumn{2}{|c|}{ Nitruro de Aluminio } & \multicolumn{2}{c|}{ Carburo de Boro } \\
\hline & $\mathrm{H}(\mathrm{GPa})$ & $\mathrm{E}(\mathrm{GPa})$ & $\mathrm{H}(\mathrm{GPa})$ & $\mathrm{E}(\mathrm{GPa})$ \\
\hline Berkovich & 13 & 343 & 34 & 545 \\
\hline Esférica & 13 & 336 & 32 & 503 \\
\hline
\end{tabular}

Es necesario indicar, que aunque los coeficientes de correlación obtenidos de los ajustes presentan valores muy cercanos a 1, los datos correspondientes a bajas profundidades de penetración se alejan de la tendencia lineal, lo cual es una consecuencia del error que se comete en la estimación del área de contacto en este rango de penetraciones, tal y como se explicó anteriormente. Además, esta desviación es aún mayor para el carburo de Boro. Por tanto, los efectos geométricos parecen ser más significativos a medida que crece la rigidez del material ensayado.

Es posible emplear una metodología similar para estimar la dureza de indentación de los materiales. La ecuación [7] relaciona la carga máxima de indentación, $\mathrm{P}$, y el área de contacto, A, a través de la dureza. En la figura 7a) se presenta la carga máxima de indentación frente al área de contacto para el nitruro de Aluminio y el carburo de Boro obtenidas a partir de ensayos realizados con indentadores Berkovich. En el nitruro de Aluminio, el coeficiente de correlación presenta un valor cercano a 1, y las dureza extraída de la pendiente de la curva es similar a la nominal. En el carburo de Boro es posible distinguir dos zonas. La parte inicial de la curva, que se corresponde con las penetraciones más pequeñas, y por tanto, es la zona que se encuentra más afectada por los fenómenos de carácter geométrico ya descritos, no presenta una tendencia lineal. Es por ello que debería desarrollarse una nueva metodología que contemple los factores influyentes anteriormente descritos. Sin embargo, cuando la profundidad de penetración es lo suficientemente elevada, el efecto de los fenómenos geométricos se disipa, y los datos experimentales se ajustan a una recta. De la pendiente de esta recta es posible obtener un valor de dureza que coincide con los valores nominales expresados en la tabla 1. En la figura $7 \mathrm{~b}$ ) se presentan las mismas representaciones para el caso de las indentaciones realizadas con indentadores esféricos. Se pueden extraer conclusiones similares a las indicadas anteriormente. Sin embargo, la zona no lineal de esta representación para el carburo de Boro es más pequeña que la que se mostraba en la figura 7a) correspondiente a los ensayos realizados con puntas Berkovich. Esto puede ser debido a que en el caso del indentador Berkovich la desviación de la geometría real con respecto a la ideal procede de la conjunción de dos factores: el redondeo de la punta y la deformación del indentador. Mientras que en el caso del indentador esférico esta desviación sólo se produce como consecuencia de un único fenómeno, que es la deformación del indentador. 

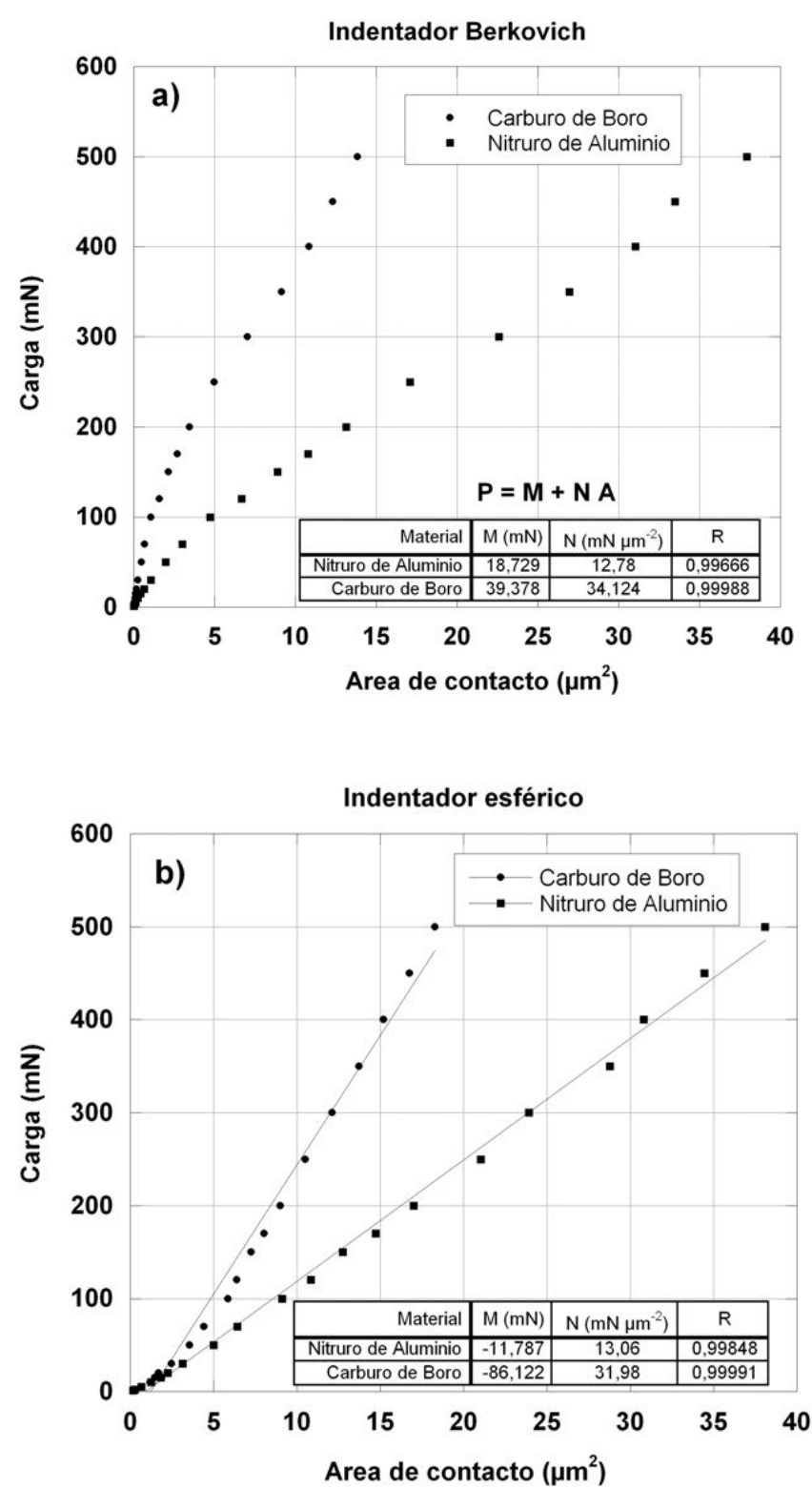

Fig. 7- a) Carga máxima de indentación, $\mathrm{P}$, frente al área de contacto, A, para los dos materiales estudiados ensayados con indentador Berkovich. b) Carga máxima de indentación, $\mathrm{P}$, frente al área de contacto, A, para los dos materiales estudiados ensayados con indentador esférico.

\section{CONCLUSIONES}

El módulo de Young y la dureza obtenidos a partir de los ensayos de indentación realizados presentan una fuerte dependencia con el tamaño de la indentación. Por tanto, no se pueden determinar las propiedades mecánicas de los materiales ensayados a partir de un único experimento. La simulación mediante elementos finitos realizada sobre un material elástico con las propiedades mecánicas del carburo de Boro ha permitido comprobar que la elevada rigidez de los materiales involucrados induce la deformación del indentador. Además, en el caso del indentador Berkovich el redondeo de la punta también puede afectar al análisis de los datos de indentación. Las desviaciones de la idealidad en la geometría del indentador que se producen como consecuencia de los fenómenos citados anteriormente, provocan una estimación errónea del área de contacto y la sobreestimación de las propiedades mecánicas calculadas. Estos efectos pueden minimizarse si se emplea una metodología basada en utilizar múltiples ensayos realizados a varias cargas máximas de indentación. Sin embargo, la influencia de los efectos geométricos descritos aumenta a medida que se incrementa la rigidez del material ensayado.

Por tanto, lo que se demuestra en este trabajo es que al indentar materiales que presenten una relación de rigidez material/indentador superior a 0,4 la incertidumbre asociada a la determinación de las propiedades mecánicas de este material es considerable; pudiendo alcanzarse diferencias cercanas al $20 \%$ con respecto al valor nominal de las mismas.

\section{BIBLIOGRAFÍA}

1. W.C Oliver, G. M. Pharr, An improved technique for determining hardness and elastic modulus using load and displacement sensing indentation experiments J. Mater. Res., 1992, 7, 1564-1583.

2. H. Hertz. On the contact of rigid elastic solids and on hardness. Verhandlungen des Vereins zur Beförderung des Gewerbefleisses, 1882, 90-156.

3. I. N. Sneddon The relation between load and penetration in axisymmetric boussinesq problem for a punch of arbitrary profile. Int. J. Eng. Sci.,1965, 3, 47-57

4. M. F., Doerner and W. D. Nix. A method for interpreting the data from depthsensing indentation instruments. J. Mater. Res., 1986, 1, 601-609.

5. W.D Nix., H. Gao. Indentation size effects in crystalline materials: A law for strain gradient plasticity. J. Mech. and Phys. of Sol. 1998, 46, 411 - 425.

6. Y. Huang, F. Zhang, K. C. Hwang, W. D. Nix, G. M. Pharr, G. Feng.A model of size effects in nano-indentation. J. Mech. and Phys. of Sol. 2006, 54, 1668 -1686 .

7. G. Feng, W. D. Nix. Indentation size effect in MgO. Scripta. Mater. 2004, 51, $599-603$.

8. K. L. Johnson. Contact Mechanics. Ed. Cambridge University Press, the Edinburgh Building, Cambridge CB2 2 RU, UK, 1985.

9. N. Yu, A. A. Polycarpou, T. F. Conry. Tip - radius effect in finite element model of sub $-50 \mathrm{~nm}$ shallow indentation. Thin solid films, 2004, 450, $295-303$.

10. J. Alkorta, J. M. Martínez - Esnaola, J. Gil - Sevillano. Detailed assessment of indentation size - effect I recrystallized and highly deformed niobium. Acta Mater, 2006, 54, $3445-3452$.

11. J. A. Knapp, D. M. Follstaedt, S. M. Myers, J. C. Barbour, T.A. Friedmann. J. Appl. Phys., 1999, 85, 1460.

12. A. Faulkner, K. C. Tang, S. Sen, R. D. Arnell. J. Strain. Anal. 1998, 33, 411.

13. S. M. Jeong, H. L. Lee. Finite element analysis of the tip deformation effect on nanoindentation hardness. Thin Solid Films, 2005, 492, 173 - 179.

14. A. Rico, M. A. Garrido, E. Otero, J. Rodriguez. Roughness effect on the mechanical properties of ceramic materials measured from nanoindentation tests. Key Eng. Mater. 2007, 333, 247 -250.

15. S. Guicciardi, A. Balbo, D. Sciti, C. Melandri, G. Pezzoti. Nanoindentation characterization of SiC - based ceramics. J. Eur. Ceram. Soc. 2007, 27, 1399 -1404 .

16. Z. Peng Z., J. Gong, H. Miao. On the description of indentation size effect in hardness testing for ceramics: Analysis of the indentation data. J. Eur. Ceram. Soc, 2004, 24, 2193-2201.

17. J. Gong, Z. Peng, H. Miao. Analysis of the nanoindentation load displacement curves measured on high - purity fine - grained alumina. J. Eur. Ceram. Soc. 2005, 25, 649 - 654 .

18. J. Gong, H. Miao, Z. Peng, L. Qi. Effect of peak load on the determination of hardness and Young's modulus of hot-pressed $\mathrm{Si}_{3} \mathrm{~N}_{4}$ by nanoindentation. Mater. Sci. and Eng., 2003, A 354, 140-145.

19. F. Gálvez. Caracterización mecánica de materiales cerámicos avanzados a altas velocidades de deformación. Tesis Doctoral. Universidad Politécnica de Madrid. Madrid (1999).

20. J. G. Swadener, E. P. George, G. M. Pharr. The correlation of the indentation size effect measured with indenters of various shapes. J. Mech. and Phys. of Sol., 2002, 50, 681 - 694.

Recibido: 05.11 .07

Aceptado: 31.03 .08 